\title{
Crianças atendidas em pronto- atendimento e pronto-socorro por causas externas: subsídios para melhoria da assistência
}

\author{
Recebido em: 08/12/2010 \\ Aceito em : 14/04/2011
}

Daniela da Silva Garcia

Trata-se de um estudo descritivo, retrospectivo, de abordagem quantitativa, sobre vítimas de causas externas menores de 10 anos, atendidas em prontoatendimento (PA) e pronto-socorro (PS). O estudo realizado nos meses de abril a julho de 2007 utilizou as informações registradas nos serviços, acrescidas daquelas obtidas por entrevista com os pais ou responsáveis legais, no domicílio. A população foi de 29 crianças menores de 10 anos e vítimas de causas externas atendidas em pronto-atendimento e pronto-socorro no município de Populina-SP. Os meninos predominaram e a causa externa mais frequente foi o acidente de transporte com ciclistas. Quanto ao local da ocorrência, o lar foi o mais frequente e a maioria ocorreu durante as brincadeiras infantis.

Descritores: Causas Externas, Informações, Acidentes, Crianças.

\section{Information on external causes in children at first aid treatment and emergency room - subsidies for its prevention}

This is a descriptive retrospective study, a quantitative approach about victims of external causes less than 10 years, treated in Emergency Department (ED) and Emergency Room (ER).The study was carried out from April to July/2007 and used recorded data in these Services plus the ones by means of interview with family of the studied children, at home. The studied population was composed by 29 children with less than 10 years old and victims of external causes attended in First Aid Treatment and Emergency Room in Populina-SP. The masculine gender predominated and the major external cause was the accident of transportation with cyclists. Concerning local where the accidents happened, home appeared in major number. The biggest part of the accidents happened during children's plays.

Descriptors: External Causes, Information, Accidents, Children.

\section{Informaciones sobre causas externas en niños atendidos en primeros auxilios y emergencias - subsidios para su prevención}

Se trata de un estudio descriptivo, retrospectivo, de abordaje y amplitud cuantitativo sobre víctimas de causas externas menores de 10 años, atendidas en Primeros Auxilios y Emergencias. La población estudiada fue de 29 niños menores de 10 años y victimas de causas externas que fueran atendidas en Primeros Auxilios y Emergencias en el municipio de Populina (SP). El sexo masculino predominó y los accidentes de transporte con ciclistas ocuparon el primer lugar. Respecto al local donde ocurrieron los accidentes, la casa apareció en mayor porcentaje. La mayor parte de los accidentes ocurrió durante los juegos las bromas infantiles.

Descriptores: Causas Externas, Informaciones, Accidentes, Niño.

\section{INTRODUÇÃO}

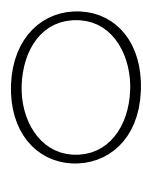
$S$ profissionais de saúde recebem em prontoatendimento (PA), pronto-socorro (PS) e Unidades Básicas de Saúde (UBS) crianças encaminhadas para atendimento, com histórias de lesões físicas e acidentes ocorridos em domicílio ou extradomicílio, como creches e escolas, os lugares mais frequentados por elas. Entre as causas que levam as crianças ao atendimento encontramse as causas externas.

Causas externas configuram-se em um conjunto de agravos à saúde, que levam à internação ou ao óbito e incluem as causas denominadas como acidentes de trânsito, quedas, envenenamentos, afogamentos, entre outros, e as causas intencionais, como agressões e lesões autoprovocadas ${ }^{(1,2)}$.
Esses agravos têm importância por ocasionarem consequências orgânicas, psicológicas, sociais e econômicas e serem responsáveis por significativa parcela da morbidade, mortalidade e sofrimentos humanos ${ }^{(2)}$.

Esse tipo de acidente ocupa as cinco principais causas de mortalidade na maioria dos países. Representam, a partir da década de 1980, a segunda causa de morte no quadro geral de mortalidade ${ }^{(3)}$, sendo responsáveis por traumatismos não fatais e sequelas que exercem grande impacto em longo prazo, repercutindo na família e na sociedade, representando importante carga social pelas vidas perdidas, pelas sequelas e por onerarem a sociedade com custos diretos e indiretos ${ }^{(3)}$, o que justifica este estudo e pode possibilitar a elaboração 
de um diagnóstico que contribua para a implementação e execução de estratégias específicas de controle e prevenção ${ }^{(4,2)}$. Atualmente, em todo o mundo, a alta incidência dos acidentes na infância, bem como a importância de seu controle e prevenção, continua despertando o interesse por estudos que possam direcionar melhor e fundamentar a implementação, a execução e a avaliação de estratégias de prevenção específicas ${ }^{(5)}$.

A criança, em virtude da imaturidade, curiosidade, crescimento e desenvolvimento, encontra-se, muitas vezes, indefesa, vulnerável e propensa aos acidentes ${ }^{(6)}$. Acidentes domésticos com crianças menores de 10 anos são situações complexas e revelam grave problema de saúde pública. Ainda é possível que existam no mundo indicadores elevados de acidentes infantis porque o acidente ainda é interpretado como obra do destino ou do acaso, ou ainda como algo comum nessa faixa etária(7).

Segundo a literatura, nos Estados Unidos, a cada ano, aproximadamente 600 mil crianças são atendidas em hospitais por causas externas, sendo que de 70 a 80 crianças morrem por essas causas $^{(6)}$. A partir do primeiro ano de vida, acidentes de transporte e quedas são as principais causas de óbitos em crianças de todas as idades; em se tratando de intoxicações, em 1999, ainda nos EUA, de um total de 873 óbitos por essa causa, 52,5\% eram crianças menores de 6 anos de idade ${ }^{(8)}$. A cada ano, 3 mil crianças morrem devido a acidentes domésticos; as queimaduras são a principal causa de óbito, seguindo-se a asfixia e o afogamento.

No Brasil, em 2000, pudemos observar que houve 4.560 óbitos resultantes de causas externas, representando um coeficiente de 13,8/1000.000 habitantes em 2001 - houve um decréscimo na mortalidade, apresentando um coeficiente de 12,7/100.000 habitantes; em 2002, um coeficiente de 12,8/100.000 habitantes; em 2003, um coeficiente de 12,5/100.000 habitantes; em 2004, 12,3/100.000 habitantes, visto que a população total de crianças menores de 10 anos do país era de 32.918.055; em 2005, o coeficiente obtido foi de $11,7 / 100.000$ habitantes, ou seja, observou-se decréscimo na $\operatorname{taxa}(9,10)$.

No estado de São Paulo, em 2000, houve 845 óbitos, e o coeficiente obtido foi de 12,3/100.000 habitantes. O total de menores de 10 anos foi de 6.861 .432 crianças. Em 2004, dentro dessa mesma faixa etária, ocorreram 621 óbitos por causas externas. O coeficiente foi de 9,1/100.000 habitantes, Verificou-se, assim, que também no estado de São Paulo esse coeficiente específico mostrou pequena queda ${ }^{(11,12)}$.

O Sistema de Informação Hospitalar do Sistema Único de Saúde (SIH/SUS) foi concebido para operar o sistema de mapeamento de internação dos hospitais próprios do SUS e contratados, e tem apresentado melhoras gradativas em suas informações ao longo de sua existência(13). Está limitado nas internações hospitalares, ou seja, exclui informações de atendidos no PS e liberados.

Em nosso meio, em geral, as vítimas atendidas têm uma ficha de atendimento preenchida na unidade de assistência e liberadas, incluindo crianças menores de 10 anos; entretanto, esses dados não estão disponíveis em sistema de informação passível de ser acessado, e as informações sobre essa parcela de vítimas no Brasil ficam incompletas, e o total delas se perde sem um meio de registro ideal e confiável(14).

Existem, no país, alguns estudos sobre crianças vítimas de causas externas atendidas em pronto-atendimento (PA) e pronto-socorro (PS) que mostram que a maioria das vítimas de causas externas, incluindo as crianças, foi atendida em pronto-socorro $^{(5,2,14)}$.

Considerando-se que, num município com cobertura total pelo PSF, seja possível identificar, utilizando múltiplas fontes de informações, os tipos de causas externas que mais acometem crianças menores de 10 anos, objetiva-se, com o presente estudo:

- identificar quantitativamente os dados das vítimas descritos nas Fichas de Atendimento Ambulatorial (FAA) e laudo de internação acrescidos daqueles posteriormente obtidos por entrevista junto aos pais ou responsáveis legais, no domicílio;

- identificar os tipos de causas externas, relacionando-os ao sexo e à idade das crianças, ao local e à circunstância do evento, à natureza da lesão e às partes do corpo atingidas.

\section{MÉTODO}

Trata-se de um estudo descritivo, retrospectivo, de abordagem quantitativa. O estudo foi realizado no município de Populina (SP) utilizando dados registrados nas fichas de atendimento de crianças menores de 10 anos, vítimas de causas externas, atendidas no pronto-socorro da Santa Casa local, pronto-atendimento (CSII), juntamente com PSF's I e II. Como os dados sobre o local e as circunstâncias do evento, em geral, não constam nas fichas utilizadas por esses serviços, posteriormente foram complementadas por entrevistas com pais ou responsáveis legais da criança em visitas domiciliárias.

O município de Populina, com menos de 5 mil habitantes, a 620 quilômetros por rodovia da capital, apresenta $100 \%$ de sua população urbana e rural cadastrada em PSF I e II13. Atualmente, o município conta com um hospital local (Santa Casa de Misericórdia de Populina), uma Unidade de Saúde (CSII) e duas Equipes de PSF (equipes I e II). As urgências são encaminhadas à Unidade de Saúde e as emergências, atendidas no PS da Santa Casa.

Para a realização do presente estudo, foram utilizados os dados registrados nas fichas das crianças, preenchidas durante o atendimento nos PA e PS devido a causas externas ou lesão 
e os dados obtidos em entrevista durante visita domiciliária.

Primeiramente, foram selecionadas as fichas que apresentaram dados referentes ao atendimento a crianças menores de 10 anos, de ambos os sexos, que possuíam endereço no município de Populina. Após essa pré-seleção, foram selecionadas aquelas que apresentaram como causa do atendimento lesões, envenenamentos e outras consequências de causas externas incluídas nos capítulos XIX e XX da Classificação Internacional de Doenças (CID-10) ${ }^{(15)}$.

A seguir, foram complementadas por entrevista com familiares, no domicílio das mesmas.

Antecedendo a coleta de dados, houve autorização formal da Secretaria de Saúde Municipal de Populina, e o projeto foi aprovado pela Comissão de Ética em Pesquisa da Universidade de Guarulhos, parecer 102/2007, cumprindo a resolução 196/96 Conep. Todos os participantes foram previamente informados sobre os objetivos da pesquisa, bem como sua participação voluntária, e assinaram o termo de compromisso e de consentimento esclarecido.

\section{RESULTADOS E DISCUSSÃO}

A população estudada foi composta de 29 crianças. A primeira fase constituiu-se da coleta de dados através da fichas de atendimento em PA e PS (incluindo as 29 crianças); a segunda fase, entrevista com as famílias das crianças (incluindo 21 crianças).

O que chama a atenção, neste estudo, é que $100 \%$ das vítimas foram liberadas, diferenciando-o dos demais aqui apresentados. Devemos ressaltar que, no município, as vítimas de causas externas "graves" em qualquer idade, inclusive as crianças, não são atendidas no PS da Santa Casa local, mas encaminhadas para o município de referência, capacitado para atendimentos de maior complexidade. Dessa forma, essas internações não constam nos registros do município em análise.

A variável "como aconteceu o acidente" foi encontrada nas fichas em $31,2 \%$ e $72,4 \%$ necessitaram da entrevista, sendo que as informações contidas nas fichas de atendimento foram obtidas para as 29 crianças ( $100 \%$ dos casos selecionados para este estudo), e as variáveis que necessitaram da entrevista foram obtidas para 21 crianças $(72,4 \%)$.

A análise dos tipos de causas externas relacionados a idade e sexo das crianças mostrou que a faixa etária mais atingida foi de 5 a 9 anos e que o sexo masculino foi o mais acometido pelas causas externas, representando $65,5 \%$ das crianças (tabela 1 ). É uma diferença relevante, que nos leva a concluir que, pelo fato de o sexo masculino aparecer em maior número, justificase seu maior risco para acidentes, talvez por apresentar uma exposição maior às causas externas e não por se encontrar em maior número.

O tipo de acidente, em sua maioria, pode ser identificado através da entrevista realizada com a família da criança.

Das 29 crianças estudadas, as informações sobre tipo de causa externa e natureza da lesão foram preenchidas em 21 dos casos (tabela 2). Pode-se observar que o tipo de causa externa que ocasionou lesões foi acidente de transporte com ciclista, representando um total de $33,3 \%$ dos casos estudados. As quedas variadas vêm a seguir, com $23,8 \%$ dos casos, juntamente com impacto acidental contra objetos ou outra pessoa e, por último, quedas de objeto sobre a pessoa e instrumento cortante/perfurante, com 9,5\%. No total, as causas externas presentes na tabela 2 , principalmente as quedas, levaram a uma maior ocorrência de cortes/perfurações e lacerações como natureza da lesão.

No estudo de Fogaça ${ }^{(14)}$, em 1.438 fichas levantadas por causas externas, as quedas aparecem com $33,5 \%$, ocupando o primeiro lugar, e acidentes de transporte, com 22,5\%, vêm logo a seguir. O estudo também mostra a ocorrência de queimaduras, agressões físicas e suicídio na população estudada, o que não ocorreu na presente pesquisa.

Em trabalho realizado em Pouso Alegre (2), foram identificadas lesões acidentais $(73,2 \%)$ e acidentes de transporte $(13,7 \%)$ como causas mais frequentes na população estudada, aparecendo também violências,

agressões e tentativas de suicídio.

Isso demonstra que os resultados podem depender do tipo de população estudada e seus costumes. Populina, em virtude de ser um município pequeno e de poucos recursos, com população de baixa renda, o meio de transporte mais utilizado entre adultos e crianças é a bicicleta, por isso o maior número de acidentes vem a ser com ciclistas.

Os acidentes na infância envolvem vários segmentos populacionais, com peculiaridades marcantes em relação à faixa etária, ao local de ocorrência e às características ou circunstâncias em que se desenvolvem ${ }^{(16)}$.

A característica que mais influenciou nos resultados foi o fato de a população utilizar-se da bicicleta como meio

Tabela 1 - Distribuição das crianças vítimas de causas externas segundo sexo e idade. Populina, 2007

\begin{tabular}{|c|c|c|c|c|}
\hline \multirow[b]{2}{*}{ Idade (anos) } & \multicolumn{2}{|c|}{ SEXO } & & \\
\hline & M & $\mathbf{F}$ & & \\
\hline & $\mathrm{N} \quad \%$ & $\mathrm{~N} \quad \%$ & $\mathrm{~N}$ & $\%$ \\
\hline Menor que 1 & 1 & - & 1 & 3,5 \\
\hline De 1 a 4 & 5 & 5 & 10 & 34,5 \\
\hline De 5 a 9 & 13 & 5 & 18 & 62,1 \\
\hline Total & $19(65,5)$ & $10(34,5)$ & 29 & (100) \\
\hline
\end{tabular}

Nota: Os números entre parênteses referem-se às \% calculadas na linha. 
Tabela 2 - Distribuição das crianças segundo tipos de causas externas e natureza da lesão. Populina, 2007

\begin{tabular}{|c|c|c|c|c|c|c|c|c|c|c|}
\hline \multicolumn{11}{|c|}{ Natureza da lesão } \\
\hline $\begin{array}{l}\text { Tipos } \\
\text { de C.E. }\end{array}$ & \multicolumn{2}{|c|}{ Fratura } & \multicolumn{2}{|c|}{$\begin{array}{l}\text { Entorse/ } \\
\text { luxação }\end{array}$} & \multicolumn{2}{|c|}{$\begin{array}{c}\text { Corte/ } \\
\text { perf./laceração }\end{array}$} & \multicolumn{2}{|c|}{ Outros* } & \multicolumn{2}{|c|}{ TOTAL } \\
\hline & $\mathrm{N}$ & $\%$ & $\mathrm{~N}$ & $\%$ & $\mathrm{~N}$ & $\%$ & $\mathrm{~N}$ & $\%$ & $\mathrm{~N}$ & $\%$ \\
\hline $\begin{array}{l}\text { Acidente de } \\
\text { transporte com } \\
\text { ciclista }\end{array}$ & - & - & 2 & 9,5 & 2 & 9,5 & 3 & 14,2 & 7 & 33,3 \\
\hline Quedas variadas** & - & - & - & - & 3 & 14,2 & 2 & 9,5 & 5 & 23,8 \\
\hline $\begin{array}{l}\text { Impacto acidental } \\
\text { contra objeto ou } \\
\text { pessoa }\end{array}$ & 1 & 4,7 & - & - & 2 & 9,5 & 2 & 9,5 & 5 & 23,8 \\
\hline $\begin{array}{l}\text { Instrumento } \\
\text { cortante/perf. }\end{array}$ & - & - & 2 & 9,5 & - & - & 2 & 9,5 & - & - \\
\hline $\begin{array}{l}\text { Queda de objeto } \\
\text { sobre a pessoa }\end{array}$ & 1 & 4,7 & - & - & - & - & 1 & 4,7 & 2 & 9,5 \\
\hline Total & 2 & $(9,5)$ & 2 & $(9,5)$ & 9 & $(42,8)$ & 8 & (38) & 221 & $(100)$ \\
\hline
\end{tabular}

*Acidentes identificados com traumas diversos. **Eeito, própria altura (como sofá e berço) e outras alturas.

de transporte mais comum e levarem crianças na garupa, ocasionando acidentes. No estudo de Mesquita $^{(2)}$, o item "outras causas externas de traumatismos acidentais" traz uma peculiaridade de sua população estudada, que é "mordedura ou golpe provocado por cão", representando 12,1\% em uma população de 380 vítimas por causas externas de um município do interior do estado de Minas Gerais, o que revela, mais uma vez, a influência das características da população estudada nas ocorrências das causas externas.

O maior número de causas externas que acometeram a população estudada ocorreu em domicílio (38\%), predominantemente frente aos demais tipos de causas externas levantadas. Os acidentes de transporte ocorreram em sua maioria na via pública (rua), em $23,8 \%$ dos casos; apareceu apenas um acidente de transporte em casa (isso significa que uma criança se machucou com a bicicleta no quintal de sua casa). O maior número de acidentes se concentrou no lar e na via pública, chamando a atenção para a importância desses dois locais e a frequência de acidentes que os mesmos proporcionam para as crianças menores de 10 anos. Assim, podemos concluir que os acidentes com crianças menores de 10 anos possuem maior possibilidade de acontecerem em locais que as crianças frequentam e onde permanecem mais tempo.

Em outros dois tipos de estudo relacionados à causas externas $^{(2,4)}$, observou-se que a residência é o local em que mais ocorrem acidentes, tal como o encontrado em nosso estudo, ficando em segundo lugar a via pública. No estudo de Fogaça(14), a via pública teve predominância. Em se tratando de estudos sobre causas externas, faz-se necessário o conhecimento do local em que essas ocorrem, para que se possa pensar em prevenção.

$\mathrm{Na}$ análise dos tipos de causas externas, verificou-se que o maior número de acidentes aconteceram quando a criança se encontrava brincando (23,8\% das situações), seguido por acidentes em que a criança estava andando na garupa de bicicleta com um adulto e colocou o pé no raio do equipamento, causando a lesão e queda de objetos sobre a criança, ambos representando $19 \%$ cada. Isso nos deixa clara a importância de sabermos como aconteceu o acidente, para conhecer a circunstância em que o mesmo ocorreu, a fim de que se possam desenvolver, com base nessa informação, formas de prevenção desses acidentes.

\section{CONSIDERAÇÕES FINAIS}

A causa externa que predominou foi acidente de transporte com ciclistas, com sete $(33,3 \%)$, e, em sua maioria, crianças levadas na garupa que acidentalmente colocaram os pés no raio da bicicleta.

As informações obtidas neste estudo são relevantes enquanto considerada a necessidade de implantação de um sistema eficaz de informações sobre vítimas de causas externas. Conhecer quais os tipos de causas externas que mais atingem essas crianças, quais as circunstâncias que as propiciam, locais onde mais ocorrem e características das vítimas possibilitam maior compreensão desses acidentes, colaborando para a realização de ações integrais de prevenção pelo setor de saúde.

É necessária a formulação de um banco de dados para registro adequado das vítimas que são atendidas em PA e PS 
e liberadas e um melhor planejamento da assistência a esses casos, valorizando e priorizando o momento do atendimento à vítima, que fornecerá informações para subsidiar ações de vigilância epidemiológica contra causas externas em crianças menores de 10 anos.

Sendo acidente de transporte com bicicleta a causa externa predominante nessa população e considerando que a maioria das vítimas se encontrava na garupa da bicicleta e colocou o pé no raio da mesma em movimento, sugerimos que a proteção em forma de rede de náilon seja fixada no pneu traseiro da bicicleta. Qualquer adulto pode confeccioná-la (é acessível e de baixo custo), como se segue:

- retirar o pneu traseiro da bicicleta;

- cortar um pedaço de "arame liso" na medida do arco do pneu e encaixar entre os raios, modelando o mesmo e fixando-o nos raios próximos à válvula do pneu;

- utilizar um recorte no formato da circunferência do pneu da bicicleta, feito de náilon transparente, denominado "sombrite", para fixar no arco de arame já colocado no pneu, deixando o espaço da válvula sem cobertura para não atrapalhar quando for preciso utilizar a bomba de ar para encher o pneu; - para fixar o recorte de náilon no arco de arame já colocado no pneu, utilizar linha de náilon própria para pesca;

- após a fixação estar completa, deixando o recorte de "sombrite" bem distendido, recolocar o pneu traseiro da bicicleta,
"Sugerimos

que a proteção

em forma de rede de náilon seja fixada no pneu traseiro da bicicleta" assim os pés da criança não vão entrar em contato com os raios em movimento, pois o "sombrite" formará uma proteção sobre os mesmos, sendo que a proteção não irá atrapalhar na manutenção do pneu nem interferir na estética do modelo da bicicleta.

O lar - onde as crianças mais frequentam, permanecem mais tempo e desenvolvem atividades rotineiras em suas brincadeiras foi o local de predominância dos acidentes em menores de 10 anos.

Esperamos que as informações obtidas neste trabalho venham a auxiliar e subsidiar a formulação de políticas públicas voltadas à prevenção de causas externas em crianças menores de 10 anos que são atendidas em PA e PS e, em seguida, liberadas.

\section{Referências}

1. Martins CBG, 1. Martins CBG, Andrade SM. Causas externas entre menores de 15 anos em cidade do Sul do Brasil: atendimentos em Pronto Socorro, internações e óbitos [Internet]. [citado em 2006 Jun 10]. Disponível em: http:// www.scielo.com.

2. Mello Jorge MHP, Latorre MRDO. Acidentes de trânsito no Brasil: dados e tendências [Internet]. [citado em 2006 Jul 07]. Disponível em: http://www. scielo.com.

3. Imperatori G, Lopes MJM. Estratégias de intervenção na morbidade por causas externas: como atuam agentes comunitários de saúde? [internet]. [citado em 2011 Abr 19]. Disponível em: http://www.scielo.com.

4. Oliveira LR. Subsídios para a implantação de um sistema de vigilância de causas externas no município de Cuiabá/MT [tese]. São Paulo (SP): Faculdade de Saúde Pública da Universidade de São Paulo; 2006.

5. Martins CBG, Andrade SM. Estudo descritivo de quedas entre menores de 15 anos no município de Londrina (PR Brasil) [Internet]. [citado em 2011 Abr 19]. Disponível em: http://www.scielo.com.

6. Organização Mundial de Saúde. Classificação estatística Internacional de Doenças e problemas relacionados à saúde, décima revisão. $2^{a}$ ed. São Paulo: EDUSP; 1995.

7. Acker JIBV, Cartana MHF. Construção da participação comunitária para a prevenção de acidentes domésticos infantis [Internet]. [citado em 2011 Abr 19]. Disponível em: http://www.scielo.com.

8. Prefeitura Municipal de Populina. Diagnóstico participativo de Populina.
Populina: Prefeitura; 2005.

9. Fundação SEADE. Informações dos municípios paulistas [Internet]. [citado em 2008 Fev 09]. Disponível em: http//www.seade.gov.br.

10. Instituto Brasileiro de Geografia e estatística. Censo demográfico de 2007. [Internet]. [citado em 2008 Fev 09]. Disponível em: http://.ibge.gov.br/home/ estatística/população/censo2007/universo.php.

11. Datasus. Informações de Saúde. Óbitos por causas externas [Internet]. [citado em 2008 Fev 08]. Disponível em: http://www.tabnet.datasus.gov.br. 12. Mello Jorge MHP, Koizumi MS. Gastos governamentais do SUS com internações hospitalares por causas externas: análise no Estado de São Paulo 2000. Rev Bras Epidemiol. 2004;7(2):228-38.

13. Prefeitura Municipal de Populina. Secretaria Municipal de Saúde. Plano diretor do município de Populina quadriênio 2005/2009. Populina: Prefeitura Municipal; 2005.

14. Fogaça LF. Informaçōes sobre vítimas de causas externas atendidas no pronto socorro e liberadas [dissertação]. Araras (SP): Universidade Guarulhos; 2007.

15. Organização Mundial de Saúde. Classificação estatística Internacional de Doenças e problemas relacionados à saúde, décima revisão. $2^{\mathrm{a}}$ ed. São Paulo: EDUSP; 1995.

16. Silva MAI. Perfil dos atendimentos a crianças e adolescentes vítimas de causas externas de morbimortalidade, 2000-2006 [Internet]. [citado em 2001 Abr 19]. Disponível em www.scielo.com. 